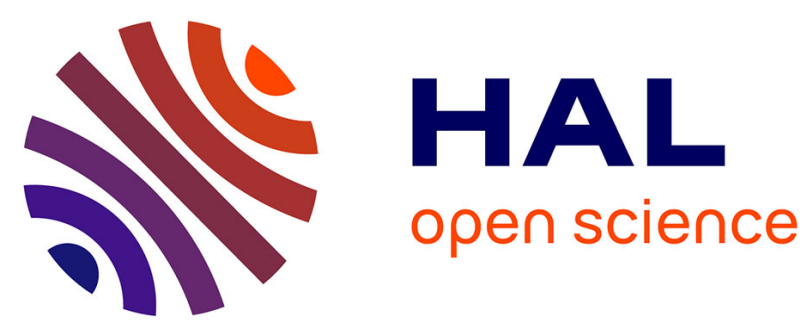

\title{
Les matériels pédagogiques pour la formation linguistique de base des résidents étrangers adultes en France : supports identitaires d'un groupe professionnel ?
}

Amélie Coulbaut-Lazzarini, Amandine Bergere

\section{To cite this version:}

Amélie Coulbaut-Lazzarini, Amandine Bergere. Les matériels pédagogiques pour la formation linguistique de base des résidents étrangers adultes en France : supports identitaires d'un groupe professionnel ?. Savoirs: Revue internationale de recherches en éducation et formation des adultes, 2012, 30 (3), 10.3917/savo.030.0059 . sic_01515902

\section{HAL Id: sic_01515902 \\ https://archivesic.ccsd.cnrs.fr/sic_01515902}

Submitted on 28 Apr 2017

HAL is a multi-disciplinary open access archive for the deposit and dissemination of scientific research documents, whether they are published or not. The documents may come from teaching and research institutions in France or abroad, or from public or private research centers.
L'archive ouverte pluridisciplinaire HAL, est destinée au dépôt et à la diffusion de documents scientifiques de niveau recherche, publiés ou non, émanant des établissements d'enseignement et de recherche français ou étrangers, des laboratoires publics ou privés. 


\title{
LES MATÉRIELS PÉDAGOGIQUES POUR LA FORMATION LINGUISTIQUE DE BASE DES RÉSIDENTS ÉTRANGERS ADULTES EN FRANCE : SUPPORTS IDENTITAIRES D'UN GROUPE PROFESSIONNEL ?
}

\author{
Amélie Coulbaut-Lazzarini et Amandine Bergere \\ L'Harmattan | Savoirs
}

$2012 / 3-n^{\circ} 30$

pages 59 à 81

ISSN 1763-4229

Article disponible en ligne à l'adresse:

http://www.cairn.info/revue-savoirs-2012-3-page-59.htm

Pour citer cet article :

Coulbaut-Lazzarini Amélie et Bergere Amandine, «Les matériels pédagogiques pour la formation linguistique de base des résidents étrangers adultes en France : supports identitaires d'un groupe professionnel ? »,

Savoirs, 2012/3 n³0, p. 59-81. DOI : 10.3917/savo.030.0059

Distribution électronique Cairn.info pour L'Harmattan.

(c) L'Harmattan. Tous droits réservés pour tous pays.

La reproduction ou représentation de cet article, notamment par photocopie, n'est autorisée que dans les limites des conditions générales d'utilisation du site ou, le cas échéant, des conditions générales de la licence souscrite par votre établissement. Toute autre reproduction ou représentation, en tout ou partie, sous quelque forme et de quelque manière que ce soit, est interdite sauf accord préalable et écrit de l'éditeur, en dehors des cas prévus par la législation en vigueur en France. II est précisé que son stockage dans une base de données est également interdit. 
Articles de recherche 


\title{
Les matériels pédagogiques pour la formation linguistique de base des résidents étrangers adultes en France : supports identitaires d'un groupe professionnel ?'
}

\author{
Amélie Coulbaut-LazZarini ${ }^{2}$ \\ et Amandine BERGERE ${ }^{3}$
}

Résumé : Le secteur professionnel de la formation linguistique de base pour adultes en France demeure flou et complexe. Cet article veut montrer que les matériels pédagogiques utilisés par les formateurs reflètent une unité, voire une identité de ce qu'il convient alors d'appeler un groupe professionnel. Basé sur les travaux en sciences du langage, mais surtout sur les travaux récents en sociologie des groupes professionnels, cet article analyse les matériels pédagogiques produits et utilisés par les formateurs. Il s'intéresse particulièrement aux principes pédagogiques et aux exercices contenus dans ces matériels, ainsi qu'à l'adéquation ou non des uns aux autres, représentatifs des tensions du secteur.

Mots clés : matériels pédagogiques, sociologie des groupes professionnels, formation linguistique de base.

Textbooks for the second language and literacy instruction of foreign residents in France: a professional group's identify?

Résumé : The French professional sector of second language and literacy instruction for adults remains unclear and complex. This article will show that the textbooks used by teachers reflect a unity, or even an identity of what could be referred to as a professional group. The article is based on

1 Contact pour l'article : amelie.coulbaut@wanadoo.fr

2 Amélie Coulbaut-Lazzarini, Titulaire d'un doctorat en STAPS (Sciences et Techniques des Activités Physiques et Sportives) de l'université Paris Descartes, chercheur à la chaire internationale Econoving, REEDS, Université Versailles-Saint-Quentin en Yvelines. amelie.coulbaut@wanadoo.fr

3 Amandine Bergère, Titulaire d'un doctorat en sciences du langage de l'université Paris Descartes, formatrice, formatrice de formateurs FLE. Co-fondatrice de l'association Va Savoirs. Directrice-adjointe de l'AEFTI Paris. amandine.bergere@gmail.com 
studies carried out in the field of linguistics, and mainly on recent research in the sociology of professional groups. It analyzes the textbooks produced and used by teachers. It focuses on educational principles and exercises contained in these books, while asking how adequately these are related, and to what extent they represent tensions within the sector.

Keywords: textbooks, sociology of professional groups, adult literacy.

\section{Los materiales pedagógicos para la formación lingüística básica de adultos residentes extranjeros en Francia: soporte identitario de un grupo profesional?}

Resumen : El sector profesional de la formación lingüística básica en Francia sigue siendo poco claro y complejo. Este artículo quiere demostrar que los materiales utilizados por los formadores dan una unidad o casi una identidad a lo que se debe llamar un "grupo profesional". Basado en el trabajo de la lingüística, y especialmente en la perspectiva de la sociología de las profesiones, este artículo analiza los materiales pedagógicos producidos y utilizados por los formadores. Son estudiados particularmente los principios educativos, los ejercicios incluidos en este material al igual que el nivel de adecuación de estos dos aspectos los cuales son representativos de las tensiones del sector de la formación lingüística.

Palabras claves: materiales pedagógicos, sociología de los grupos profesionales, formación lingüística básica.

\section{1 - Introduction}

Dans cet article, nous tenterons, au travers de l'étude des matériels pédagogiques qu'il utilise, de comprendre l'identité du groupe professionnel œuvrant à la formation linguistique de base des résidents étrangers. V. Leclercq (2004) a étudié la professionnalisation des intervenants de la formation de base. Elle souligne qu'une des difficultés méthodologiques dans l'analyse de ce secteur professionnel réside dans ses contours flous, changeant au gré des politiques publiques. Pour nous aussi, l'entreprise est complexe, d'autant plus que, depuis leurs débuts, les actions de formation ont essentiellement été prises en charge par des bénévoles, une partie d'entre eux seulement accédant à emploi rétribué à partir des années 1980 (Leclercq, 2004 ; Adami, 2009). 
Jusqu'à une période récente, aucun diplôme n'était nécessaire pour exercer en tant que salarié ou en tant que bénévole.

Cette impression est certainement due au fait que le secteur qui nous intéresse s'est constitué à la croisée de divers autres. Les premiers « cours d'alpha » (alphabétisation) sont nés dans les années 1960 au sein des mouvements militants d'éducation populaire, de lutte contre l'exclusion et pour les droits des travailleurs. À partir des années 1970, avec la montée du chômage et la découverte des Français illettrés, ces actions ont rejoint celles de la formation continue, ce qui explique certains termes en usage aujourd'hui, comme « stagiaire » pour parler des apprenants ou «formateur» pour désigner les enseignants. Au sein de la formation continue, ces actions relèvent plus particulièrement de la formation linguistique de base pour les «bas niveaux de qualification ». De fait, une grande partie des termes utilisés au fil du temps pour désigner tout ou partie des activités (" alphabétisation », « remise à niveau », « lutte contre l'illettrisme ») pointent du doigt une spécialisation dans la formation d'adultes peu ou pas familiarisés avec l'écrit, qu'ils soient étrangers ou non, francophones ou non.

Les évolutions de ces dix dernières années ont également renforcé le lien que ce secteur entretenait, de longue date ${ }^{4}$, avec celui du français langue étrangère (désormais FLE) traditionnellement plutôt tourné vers la diffusion de la langue française hors des frontières nationales (les Alliances françaises et les lycées français à l'étranger). En effet, au cours des années 1970, fleurissent les publications de linguistes et leurs actions de formation de formateurs, une période dont on peut dire qu'elle se clôt avec la première publication de la grande étude transnationale sur la langue des migrants (Perdue, 1984). À partir du début des années 2000, on assiste à la mise en place d'une politique d'accueil des résidents étrangers en situation légale assortie d'un volet de formation linguistique à leur intention. Ce nouveau dispositif d'accueil et de formation a généré une production éditoriale sans précédent de matériels pédagogiques à destination des résidents étrangers chez les éditeurs phares du FLE. Parallèlement, à partir de 2005, la possession d'un diplôme de FLE devient incontournable pour les formateurs salariés de ces formations, tandis que le diplôme élaboré pour sanctionner le niveau de français atteint par les apprenants (Beacco et al., 2005) s'inscrit dans la continuité des diplômes FLE. Dans une certaine mesure, cette orientation s'est encore accentuée avec, tout récemment, la publication du Référentiel FLI Français Langue d'Intégration (Vicher, 2011).

4 Voir, par exemple, le chapitre consacré aux «travailleurs migrants et leurs familles » dans Un niveau-senil (Coste et al., 1976). 
Malgré ces apports et appartenances diverses, nous montrerons qu'autour de ce plus petit dénominateur commun que constitue la formation linguistique de base des résidents étrangers, le secteur se définit autour d'éléments relativement stables dans le temps.

Les destinataires finaux des actions qui nous intéressent sont désignés ici par les termes « résidents étrangers ». Ils renvoient à des personnes de nationalité étrangère vivant sur le sol français en situation légale ou non. Ils nous semblent mieux convenir que ceux de « migrant» ou d'« immigré». En effet, le premier, " migrant », a été largement utilisé dans les premiers travaux de sciences du langage sur les résidents non francophones (par ex. Blot et al., 1978 ; Porcher, 1982). Il est repris aujourd'hui en sciences du langage (Adami, 2005, 2008, 2009) ou en sciences de l'éducation (Leclercq, 2010). Il renvoie cependant sémantiquement à une migration temporaire de travail caractéristique des années antérieures à la décolonisation, plutôt qu'à un projet d'installation en France. Or c'est, entre autres, ce projet d'installation qui détermine les contenus de formation du secteur aujourd'hui. Par ailleurs, le second terme, "immigré », renvoie à une catégorie utilisée en démographie et qui regroupe, pêle-mêle, étrangers possédant un titre de séjours et Français naturalisés (HCI, 2002, p. 11), voire leurs enfants. C'est pourquoi nous avons opté pour un vocable plus descriptif de la situation des bénéficiaires des actions qui nous intéressent.

Mais l'entrée que nous avons choisie pour comprendre les évolutions de ce secteur est celle des intervenants, les formateurs salariés et bénévoles, et plus particulièrement des matériels pédagogiques qu'ils produisent et utilisent pour mener à bien leurs actions. Par matériel pédagogique, nous entendons ici les ouvrages utilisés par les formateurs, l'« ensemble des objets et des appareils qui aident l'enseignant à présenter des notions, des faits ou des expériences, et qui favorisent l'apprentissage des élèves $»^{5}$. Ces objets sont en effet, en tant que production de groupe et à destination du groupe professionnel, des révélateurs de son organisation, voire de son identité (Castells, 1999, p. 17). Nous en faisons une voie d'entrée pour un état des lieux d'un métier et, au-delà, d'un groupe professionnel.

\section{2 - Cadre théorique}

Louis Porcher (1987) a démontré de quelle manière la diffusion du FLE constituait l'objet d'un champ et en a défini les acteurs, les enjeux et les positions. À notre tour, nous nous proposons de dresser le portrait d'un secteur

5 CNRS, arrêté du 27 août 1992. 
dédié à l'enseignement de la langue française. Notre approche cependant ne s'inscrit pas dans la perspective bourdieusienne adoptée par Louis Porcher, puis par Fabrice Barthélémy (2007) dans son sillage. Nous nous plaçons en effet dans le cadre théorique de la sociologie des groupes professionnels (Dubar et Triper, 2005) où les méthodologies d'enseignement et les pratiques formatives viennent informer la question de l'action du formateur, lui-même acteur négociant sa place au sein d'un système.

Comment les matériels pédagogiques utilisés par les formateurs informent-ils sur le groupe professionnel du secteur de la formation linguistique de base des résidents étrangers, ses caractéristiques, ses manières d'agir, ses valeurs et permettent-ils de l'identifier? C'est la sociologie des groupes professionnels qui nous fournit cette articulation (Vézinat, 2010). Ce courant établit en effet la synthèse entre la sociologie des professions, fonctionnaliste, s'intéressant davantage au système (les dispositifs, les institutions, les réseaux), et le courant interactionniste, s'intéressant de plus près à l'acteur (son équation personnelle ${ }^{6}$ ) et à ses actions (les méthodologies d'enseignement et les pratiques formatives).

De ce fait, l'étude des matériels pédagogiques, en tant que supports formalisés de l'action formative, constitue le noyau central de la compréhension du groupe professionnel agissant dans le secteur de la formation linguistique de base pour adultes résidents étrangers. Or nous voulons montrer que les matériels pédagogiques utilisés par les formateurs reflètent une unité, voire une identité de ce qu'il convient d'appeler un groupe professionnel.

Dans ce cadre, et après une première lecture du corpus, nos hypothèses de départ sont les suivantes :

- malgré une apparente hétérogénéité des objectifs et des niveaux, les matériels pédagogiques présentent un corps cohérent de types d'exercices et de principes pédagogiques, correspondant à un groupe professionnel cohérent ;

- les principes pédagogiques et les types d'exercices qui y sont associés ne sont pas toujours en parfaite adéquation, reflétant les tiraillements entre valeurs et agir professionnel du groupe ${ }^{7}$.

6 Caractéristiques propres à une personne.

7 Cette hypothèse, dont le lecteur peut se demander ce qui a conduit à sa formulation, provient en partie d'une réflexion appuyée sur des entretiens avec des formateurs et/ou des auteurs de matériels pédagogiques, menés pour certains de manière informelle, et qui ont poussé les auteurs à chercher si une cohérence existait toujours entre les discours présentant le matériel et les exercices contenus. 


\section{3 - Cadre méthodologique}

Une précédente phase de travail ${ }^{8}$, qui s'est déroulée entre 2008 et 2009, avait permis de constituer un corpus représentatif de matériels pédagogiques utilisés pour la formation linguistique d'adultes, grâce à un recensement des matériels pédagogiques présents dans quinze bibliothèques publiques ou privées en Île-de-France. Notre analyse ${ }^{9}$ se centre ici sur les matériels consacrés à la formation linguistique de base des résidents étrangers. Nous sommes arrivés à un total de 668 matériels pédagogiques (Lehoussel et Bergère, 2010). Au sein de l'ensemble ainsi constitué, nous avons sélectionné, à des fins d'analyse représentative, les matériels pédagogiques que nous avions rencontrés le plus fréquemment. Nous avons obtenu un total de 64 matériels pédagogiques composant 41 ensembles pédagogiques, c'est-à-dire des groupements de matériels pédagogiques dont les contenus s'articulent de façon explicite dans la présentation par les concepteurs.

Deux modes d'entrée dans le corpus textuel ont été choisis : d'une part, la prise en compte des préfaces, porteuses des principes pédagogiques énoncés par les auteurs ; d'autre part, les exercices présents dans la suite des ouvrages. Pour l'analyse des types d'exercices, un relevé selon une grille de types d'exercices a été effectué ; pour l'analyse des principes pédagogiques contenus dans les préfaces, un relevé à partir d'une grille de sélection des contenus a été réalisé. Les deux grilles ont été élaborées à partir de grilles préexistantes ${ }^{10}$. Ce procédé permet une première approche des matériels pédagogiques qui limite le temps de saisie des textes.

Une fois les contenus des préfaces et les types d'exercices existants repérés grâce à nos grilles, nous avons utilisé deux logiciels spécifiques pour guider notre analyse. L'un a guidé l'analyse des types d'exercices, l'autre celle du contenu des préfaces.

8 Consultable à l'adresse suivante : http:/ /www.va-savoirs.org/ressources/

9 Cette étude a bénéficié du cofinancement du Fonds Européen d'intégration, de la Direction de l'Accueil de l'Intégration et de la Citoyenneté, de la Région Île-de-France, du Fonds régional de développement de la vie associative, de la Ville de Paris, de la Fondation Orange et de la campagne Demain Le Monde.

10 Bertoletti, M. C. et Dahlet, P. (1984) ; Cordier-Gauthier, C. (2002) ; Fonds d'action sociale (1996); Germain C. (1993); Observatoire national de la lecture (2003, 2007); Puren, C. $(2000,2001)$. 


\section{4 - Logiciels retenus : Question Data et Alceste}

Nous utilisons deux logiciels pour traiter nos données, un logiciel de traitement d'enquêtes quantitatives et de statistiques, et un logiciel d'analyse textuelle.

Le premier, Question Data, nous a servi à repérer les éléments significatifs de notre corpus de matériels pédagogiques, c'est-à-dire à identifier les exercices caractéristiques de méthodologies d'enseignement. Nous avons choisi de croiser les types d'exercices issus de notre corpus avec les dates d'édition des manuels, afin d'observer s'il existe des variations repérables dans le temps. Nous avons choisi pour cela des intervalles de dix ans, ce qui nous permet d'obtenir des résultats exploitables, un intervalle plus court dispersant les données, et un intervalle plus long ne permettant pas de repérer des différences.

Le logiciel Alceste permet d'étudier les caractéristiques sémantiques d'un texte et d'élaborer des classes de discours, en prenant en compte des variables de notre choix. Le volume de texte du corpus, lors d'une analyse avec Alceste, est réparti dans différentes classes de discours, selon un processus automatique qui repère les grandes oppositions se dégageant du texte. Le texte est découpé en UCE (unités de contexte élémentaires), c'est-à-dire des morceaux de textes que le logiciel analyse. La répartition de ces UCE va constituer ce que l'on nomme les classes de discours. Chaque classe de discours regroupe un certain nombre de mots, appartenant à un monde lexical éloigné de ceux des autres classes de discours. Max Reinert (1993) explique que «le locuteur au cours de son énonciation investit des mondes propres successifs et ces lieux, en imposant leurs objets, imposent du même coup leur type de vocabulaire. En conséquence, l'étude statistique de la distribution de ce vocabulaire devrait pouvoir permettre de retrouver la trace des "environnements mentaux" que le locuteur a successivement investis, trace perceptible sous forme de "mondes lexicaux". »C'est donc pour faire apparaître ces mondes lexicaux que nous lançons l'analyse automatisée sur notre logiciel. Afin d'éviter toute méprise, il convient de préciser ici que le logiciel, si perfectionné soit-il, n'est qu'un outil qui met en évidence notamment un type et une distribution du vocabulaire contenu dans notre corpus. L'interprétation de ces éléments demeure l'apanage du chercheur. Ce logiciel nous a servi pour l'étude des préfaces des ouvrages de notre corpus. Nous avons choisi comme variables le titre de l'ensemble pédagogique et la date d'édition des manuels. Cela nous a permis entre autres d'observer l'impact de la date sur les objectifs présentés dans ces préfaces. Il nous a également 
servi à croiser les discours issus des préfaces avec les exercices présents dans les manuels, pour révéler l'adéquation ou non des seconds aux premiers.

\section{5 - Un secteur professionnel en construction, déconstruction et... reconstruction?}

Dans le contexte institutionnel très mouvant que nous avons décrit plus haut, marqué par les changements des politiques publiques et la pauvreté des subsides, les acteurs du secteur ont-ils réussi à construire des principes d'action et un agir professionnel cohérent, qui confèrent une unité au secteur? Comment les matériels pédagogiques l'expriment-ils ? Nous présentons ici l'analyse des préfaces et des types d'exercices des matériels pédagogiques, qui nous donne une réponse à ces questions.

\subsection{Les bornes de la sphère d'action}

Lors du recensement des matériels pédagogiques, nous avons constaté que chaque nouveau site de collecte amenait un nombre très élevé de matériels pédagogiques inconnus. En effet, le corpus de matériels pour la formation linguistique de base présente une caractéristique : une très forte dispersion. Les 668 matériels pédagogiques recensés relèvent de plusieurs secteurs consacrés à l'apprentissage du français : la formation linguistique de base des adultes (FLB), dont la lutte contre l'illettrisme ; le français langue étrangère (FLE), dont le français à visée professionnelle et le français sur objectif spécifique; le français langue maternelle (FLM) à destination des scolaires et des adultes, ainsi que quelques matériels utilisés dans le cadre de l'orthophonie.

Les 64 ouvrages les plus fréquemment rencontrés (de 3 à 9 fois), qui forment le noyau central des matériels pédagogiques des formateurs, sont peu nombreux $(22 \%)$, tandis que ceux rencontrés une fois seulement constituent $60 \%$ du recensement. Les $18 \%$ restants représentent les matériels présents deux fois. Les intervenants travaillent donc avec un petit nombre de matériels pédagogiques «fondamentaux » (constituant les matériels considérés comme relevant de la formation linguistique de base), que chacun enrichit avec toute une gamme de matériels pédagogiques périphériques.

Lorsqu'on examine de plus près les titres des matériels pédagogiques, il devient évident que les matériels de très haute fréquence (cf. exemples en annexe) sont ceux qui s'adressent spécifiquement aux adultes peu ou pas scolarisés et/ou peu ou pas francophones, résidant en France. La couronne de matériels pédagogiques rencontrés une à deux fois dessine l'image d'un secteur professionnel emprunteur, qui va puiser ailleurs dans des secteurs proches de l'enseignement du français. 
Les mouvements d'oscillation politiques du secteur semblent donc avoir été intégrés par les intervenants. Ils y ont construit un "noyau stable », centré sur l'objet « langue française à destination des résidents étrangers » et de plus larges ressources pédagogiques : des matériels pour l'enseignement du français qui ne sont pas destinés à leurs publics a priori, mais qui nourrissent leurs pratiques.

\subsection{Aspects généraux sur les matériels pédagogiques}

Une étude plus large du secteur de la formation linguistique de base (Coulbaut-Lazzarini, 2012) a montré que l'idée même de conception pédagogique ou d'approche théorique était inexistante chez les formateurs rencontrés. De même, il s'avère difficile d'en trouver trace dans les matériels pédagogiques de notre corpus. Il s'agit là d'une première caractéristique surprenante de ce secteur. C'est pourquoi nous avons scruté en profondeur le contenu même des ouvrages, pour tenter de distinguer des caractéristiques définissant un groupe professionnel qui pour l'heure semble fonctionner sans support théorique, ni même de conception pédagogique.

\subsection{Les types d'exercices: un secteur qui se construit par le biais de l'emprunt}

L'analyse des types d'exercices des 41 ensembles pédagogiques pour la formation linguistique de base les plus fréquemment rencontrés montre qu'il existe une constante forte : la centration sur les compétences écrites (production et réception écrites, morphosyntaxe et sémantique, en gris lisse sur les graphiques) qui supplantent tous les autres types d'exercices (figure 1 ci-dessous) et constituent 70 à $90 \%$ des types d'exercices relevés selon la décennie où ils ont été édités (figure 2 ci-dessous).

Cette caractéristique générale doit cependant être nuancée par l'analyse plus spécifique de chacun des ensembles pédagogiques. En effet, on observe bien un fond incompressible d'exercices consacrés à l'enseignement de l'écrit, mais également des strates successives de types d'exercices complémentaires qui viennent s'y ajouter en proportions variables (figure 3 ci-dessous) ${ }^{11}$.

11 L'intérêt de ce graphique, dont la lecture peut sembler difficile en l'absence de quadrichromie, réside surtout dans la visualisation de grandes masses (en gris foncé lisse), révélant la prédominance d'un type d'approche. 

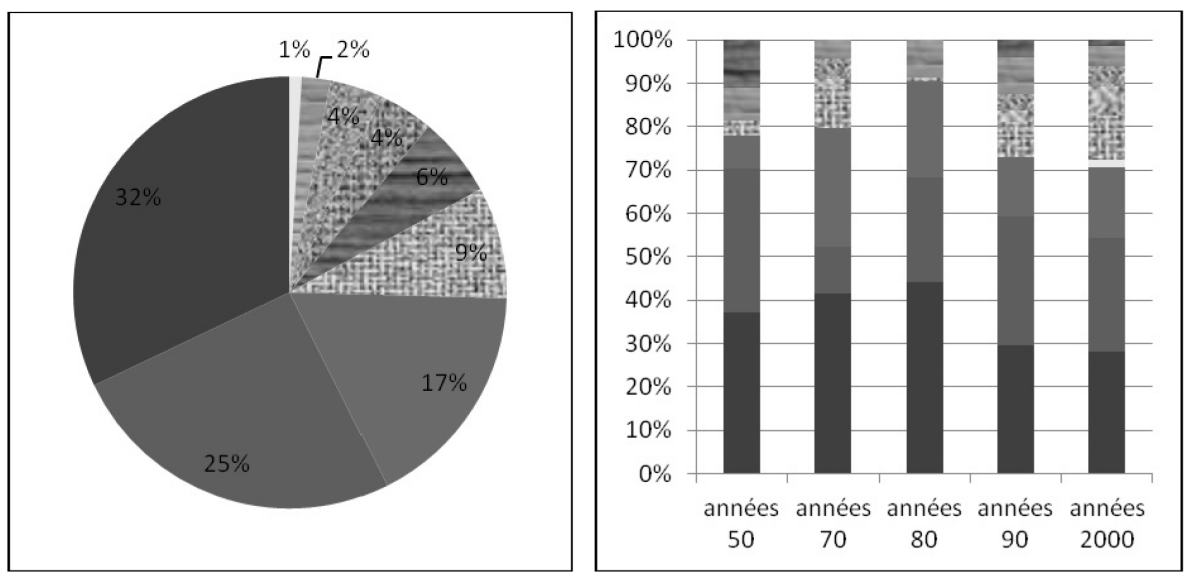

- production écrite

réception écrite

morphosynt et sém.

Exispression orale

Icorrection phonétique Ecompréhensionorale

littéracie numérique

Пopérations cognitives

ᄃ tests et évaluations

Figure 1. Répartition des grandes catégories d'exercices dans le corpus de matériels pedagogiques
Figure 2. Répartition des grandes catégories d'Exercices par décennies

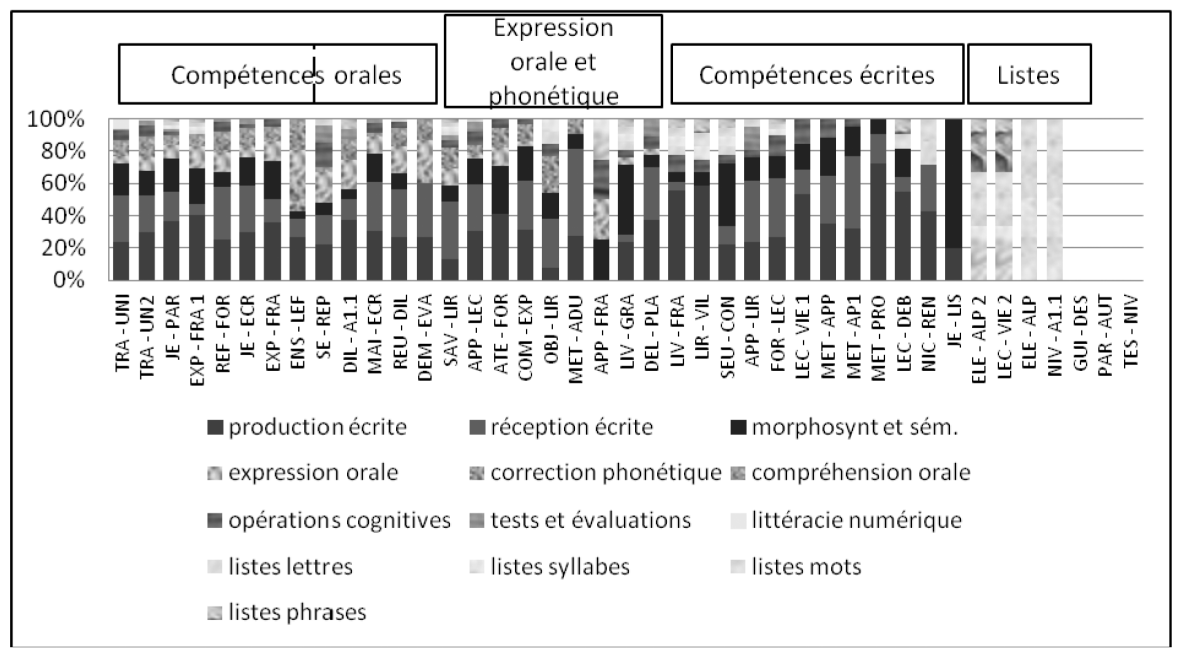

Figure 3. Répartition des grandes catégories d'exercices dans chaque ensemble pédagogique 
À l'exception de trois ensembles pédagogiques sans exercices ${ }^{12}$, les ensembles aux types d'exercices les moins variés - souvent les plus anciens présentent, a minima, des listes de lettres, de syllabes, de mots ou de phrases (vingt ensembles pédagogiques, dont quatre composés uniquement de listes, en marbré). On peut supposer, en l'absence de consigne explicite, que ces listes sont destinées à la lecture. Viennent ensuite, presque toujours, des types d'exercices consacrés à la production et à la réception écrites (32 ensembles, en gris foncé lisse), exercices de morphosyntaxe et sémantique, qui aident à l'acquisition de l'orthographe. De même, 25 ensembles pédagogiques présentent (en gris rayé) des types d'exercices destinés à travailler les opérations cognitives (par ex. « classer»).

À côté de ces types d'exercices caractéristiques de l'enseignement du français langue maternelle, on constate que les exercices consacrés à l'oral sont relativement bien représentés ( 22 ensembles pédagogiques, en vert). Mais ce sont les exercices d'expression orale et écrite qui prédominent. Ceux qui sont consacrés à la compréhension orale ne s'y ajoutent qu'assez tardivement, et ne figurent que dans 13 ensembles pédagogiques. Or ces derniers uniquement sont caractéristiques de l'enseignement des langues vivantes, et plus précisément dans le cas qui nous intéresse, du FLE. On voit là une influence relativement tardive du secteur FLE dans la typologie des exercices.

Cet effet « mille-feuille » que nous constatons est révélateur d'un savoir constitué par strates successives, comme le révèle l'analyse du nombre de types d'exercices par décennie (figure 4 ci-dessous). En effet, le nombre de matériels pédagogiques pour la formation de base chute depuis le milieu des années 1990, mais le nombre de types d'exercices, lui, ne cesse d'augmenter. Il a été multiplié par vingt entre les années 1950 et les années 2000. De façon évidente, ce secteur se constitue à vive allure et par emprunts successifs un savoir-faire spécifique en matière de formation linguistique de base des résidents étrangers.

12 Le Dictionnaire de didactique du français langue étrangère et seconde (Cuq, 2003) définit « exercice» comme "l'ensemble des travaux d'apprentissage linguistique et communicatif ». Cette définition ne nous permettant pas de savoir comment identifier un exercice, nous avons choisi de considérer qu'il y avait exercice à partir du moment où il y avait consigne. Nous définissons la consigne comme une instruction courte indiquant le type d'action que doit réaliser l'apprenant à partir d'un support sémiotique donné dans le cadre de la formation. 


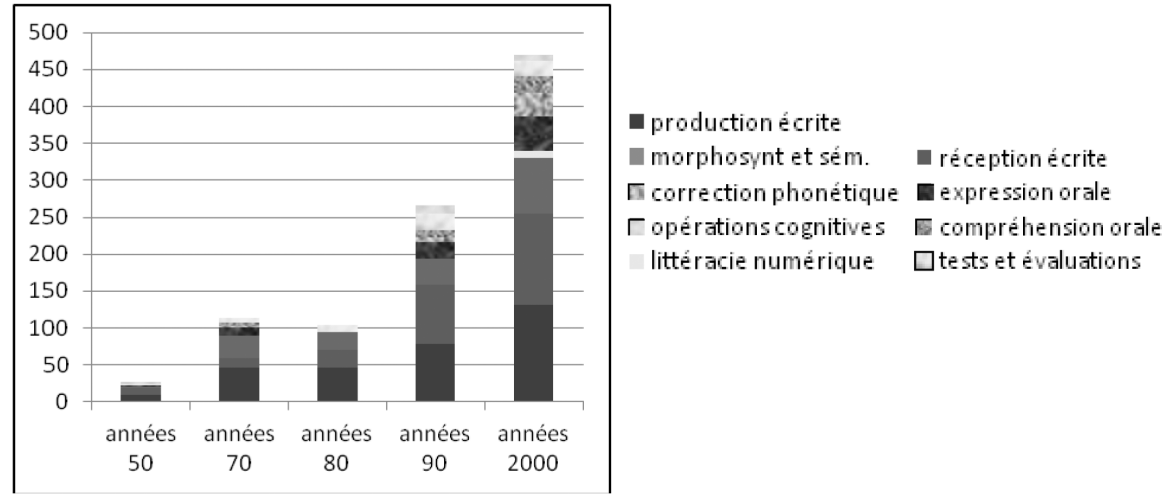

Figure 4. Evolution du nombre de types d'exercices par décennie

Dès lors, le renouveau éditorial des années 2000, marquées par la publication coup sur coup de plusieurs ensembles pédagogiques chez des spécialistes de l'édition du secteur du FLE ${ }^{13}$, peut s'interpréter tout autant comme une légitimation du savoir-faire du secteur de la formation linguistique de base par le champ du FLE, que comme une forme de capitalisation-bilan du secteur de la formation linguistique des résidents étrangers à une période où les éditeurs du FLE commencent à s'y intéresser.

En effet, dès 2003, le contrat d'accueil et d'intégration et son volet de formation linguistique pour les étrangers primo-arrivants est expérimenté (Bergère, 2008). En délimitant un marché identifiable d'apprenants, il a ouvert les portes des éditeurs phares du FLE aux experts de la formation linguistique de base des résidents étrangers. Ceux-ci ont saisi cette opportunité pour rassembler les savoir-faire du secteur. Dans notre corpus, l'exemple le plus saisissant est celui de Trait d'Union (siglé TRA-UNI et TRA-UN2 dans la figure 5 ci-dessous) : une série d'ensembles pédagogiques protéiformes, conçus en équipe, écrite par une constellation d'auteurs et rassemblant une quantité impressionnante de types d'exercices. Ces ensembles atypiques du point de vue de leur format, s'avèrent rassembler le savoir de décennies de recherches empiriques. Les autres matériels pédagogiques de ce type, édités

13 D’après Cuq et Gruca (2003), quatre éditeurs spécialisés s’inscrivent dans le champ du FLE. Parmi leurs publications pour la formation linguistique des résidents étrangers, on compte, chez CLE international, les ensembles pédagogiques Trait d'union 1 (2004), Trait d'union 2 (2005) et Trait d'union alphabétisation (2008), ainsi que DILF A1.1 150 activités (2008) ; aux Presses universitaires de Grenoble Je lis, j'écris le Francais (2004) et Je parle et je pratique le francais (2005); chez Hachette FLE l'ensemble pédagogique Savoir lire au quotidien (2005) ; et enfin, chez Didier - FLE, Réussir le DILF (2009). 
et utilisés après la constitution de notre corpus, confirment la tendance du secteur à vouloir rassembler les fils des décennies précédentes.

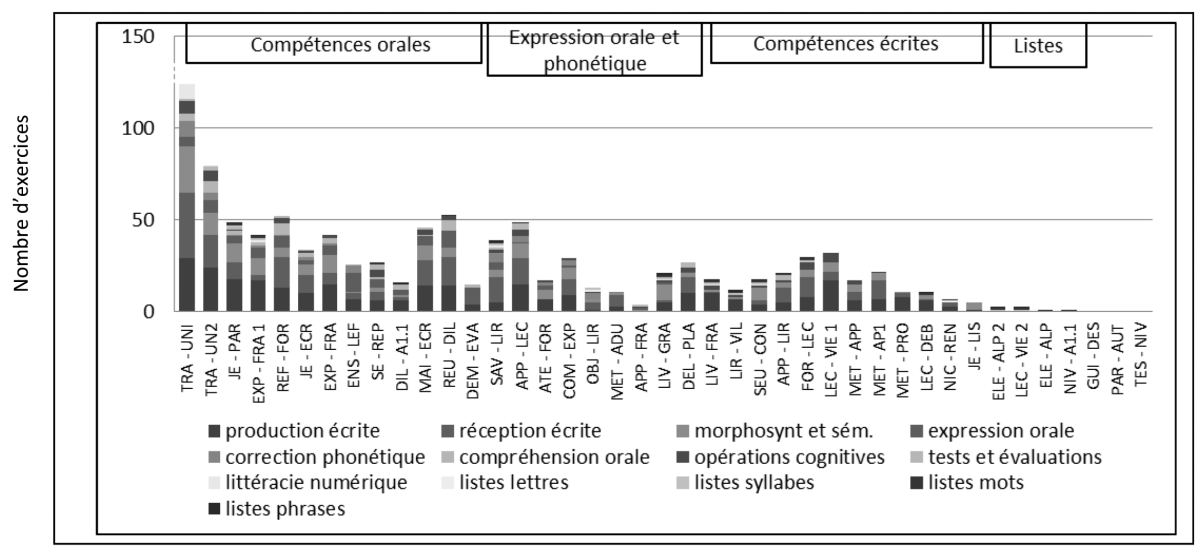

Figure 5. Nombre de types d'exercices dans chaque ensemble pédagogique (FLB)

Ces résultats, mis en parallèle avec ceux dégagés au travers de l'analyse des fréquences d'apparition des matériels pédagogiques, dépeignent un secteur professionnel en train de constituer son répertoire d'outils. Ce processus passe par le biais à la fois de la création propre de matériels spécifiques à la formation linguistique de base des adultes et par le biais de l'emprunt aux autres secteurs d'enseignement du français tels que le FLE ou le FLM.

Toutefois, il serait faux de dire que ce mouvement est arrivé à maturité. Les analyses fournies par le logiciel Alceste à partir des préfaces des matériels pédagogiques et des types d'exercices sont à cet égard extrêmement informatives.

\subsection{Les discours des préfaces, révélateurs d'une dichotomie}

L'analyse des préfaces pour elles-mêmes a dégagé deux classes de mots. La première (figure 6 ci-dessous) rassemble des termes définissant les objectifs de la formation.

Les ensembles pédagogiques associés à ces termes sont le Référentiel de formation linguistique de base, Trait d'Union, Objectif lire, Formation lecture, Maîtriser les écrits du quotidien, les Tests de niveau de départ, Programme de Base - Évaluation et enfin, le Guide descriptif des ASL. Le vocabulaire de cette classe se situe du côté de l'action, de l'utilité, de la compétence et de la maitrise du langage pour les apprenants. 
Les dates associées à ces termes et à ces ensembles pédagogiques sont très concentrées et vont de 1990 à 1998. On perçoit là une période structurante du point de vue méthodologique, marquée par l'apparition de référentiels de compétences, de programmes et de matériels pédagogiques destinés à l'évaluation orale et écrite des apprenants. Du côté des politiques publiques, on sait que le milieu des années 1990 a été marqué par la réforme des critères d'attribution des subventions pour la formation linguistique par le Fonds d'action sociale pour les travailleurs immigrés et leurs familles, principal financeur du secteur. Il semble donc que ces restructurations systémiques aient sinon provoqué, du moins été conduites dans le même mouvement que celui de formalisation des pratiques des acteurs.

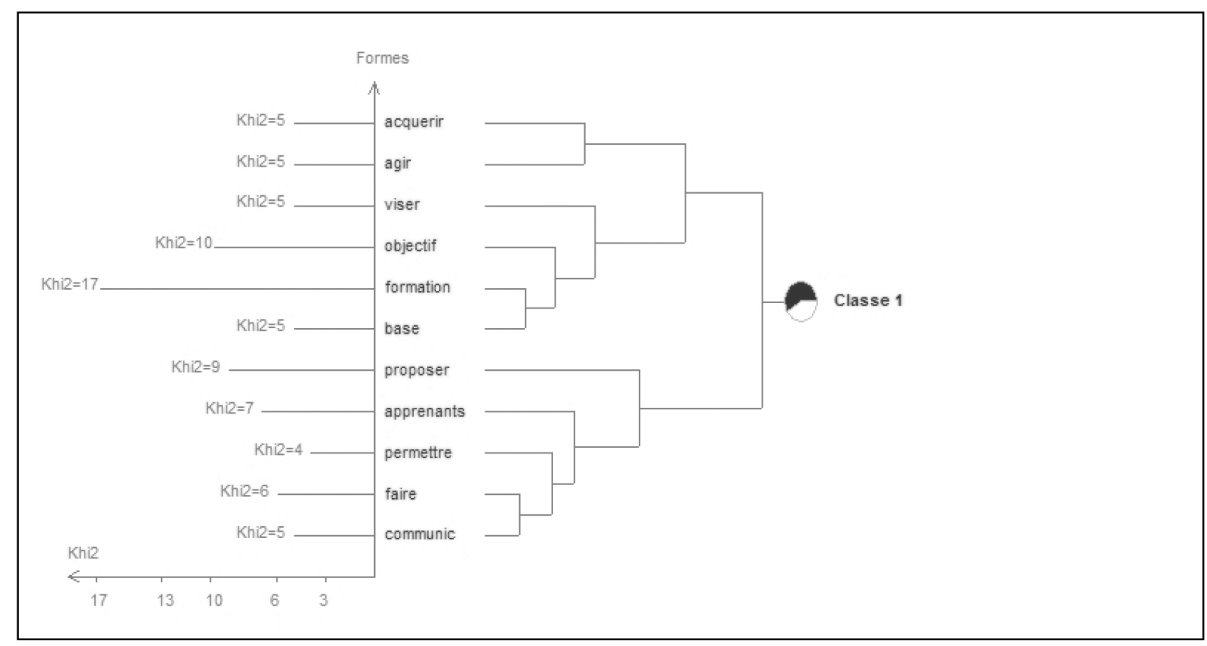

Figure 6. Première classe de termes regroupés par ALCESTE dans le corpus de préfaces de matériels pédagogiques (FLB)

On constate cependant dans la classification hiérarchique ci-dessus que les termes «formation » et «base » sont très éloignés des termes « acquérir » et «agir », et encore plus éloignés de « faire » et « communiquer». En outre, parmi les absences significatives de cette classe, c'est le terme " français » qui vient en premier lieu. On peut alors se demander comment les principes pédagogiques s'articulent réellement avec l'agir professionnel.

De fait, la seconde classe dégagée par le logiciel d'analyse sémantique rassemble des termes caractérisant les actes, les outils et les objets de la situation de formation : «lire, écrire, français langue, étranger(ère), livre, travail » (figure 7 ci-dessous). 


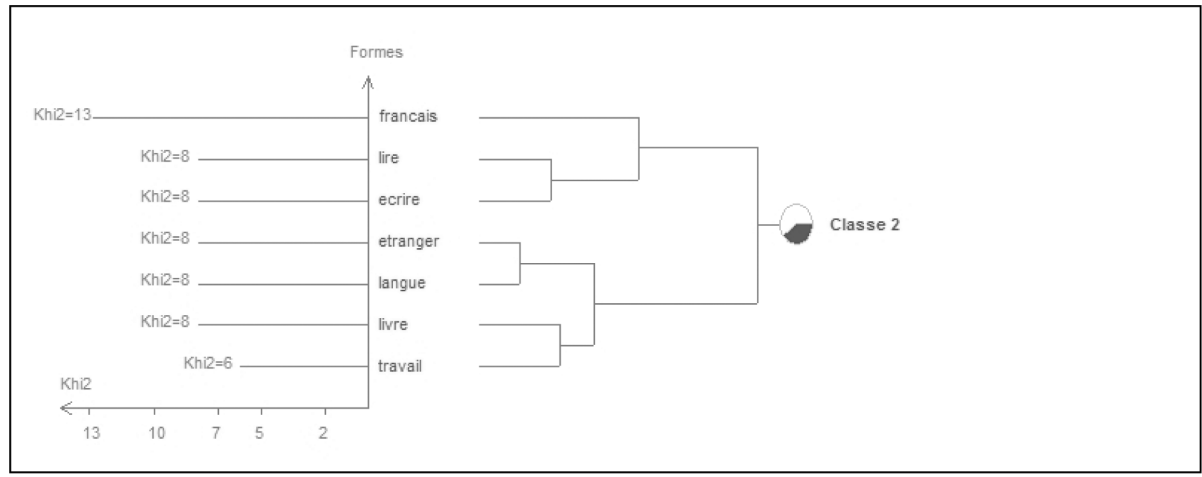

Figure 7. Deuxième classe de termes regroupés par ALCESTE dans le corpus de préfaces de matÉriels pÉdagogiques (FLB)

Les ensembles pédagogiques associés à ces termes sont Atelier d'écriture pour la formation d'adultes, Éléments d'alphabétisation, Livre de français pour les travailleurs immigrés et Savoir lire au quotidien. Les dates associées à ces termes et à ces ensembles pédagogiques sont dispersées dans la période considérée : 1972 et 1975 d'une part et 2000 d'autre part. On perçoit là la continuité des contenus sur une longue période de temps, avec une grande focalisation sur la lecture et l'écriture. Cependant, parmi les absences significatives de cette classe, c'est le terme «formation » qui vient en premier lieu. Or c'est bien dans la période qui se situe entre les années 1980 et la fin des années 1990 que le secteur s'est le plus identifié à celui de la formation professionnelle.

En d'autres termes, il ressort de l'analyse sémantique des préfaces des matériels pédagogiques que les objectifs de la formation (classe 1) sont dissociés des moyens de la formation (classe 2). Il apparaît que les objectifs de la formation sont plutôt issus l'identification longue du secteur à celui de la formation continue, mais que les moyens de l'action viennent d'ailleurs et que le dialogue entre ces deux éléments nécessaires à l'agir professionnel n'ait pas encore trouvé une articulation propre.

Ce résultat est encore affiné par le croisement des données issues de préfaces avec celles issues des exercices. Alceste dégage cinq classes de discours de cette analyse croisée. Cet arbre (figure 8 ci-dessous) correspond à une classification descendante hiérarchique, c'est-à-dire une segmentation du discours en mondes lexicaux différenciés, qui fait apparaitre les oppositions de vocabulaire qui existent dans les textes étudiés. 


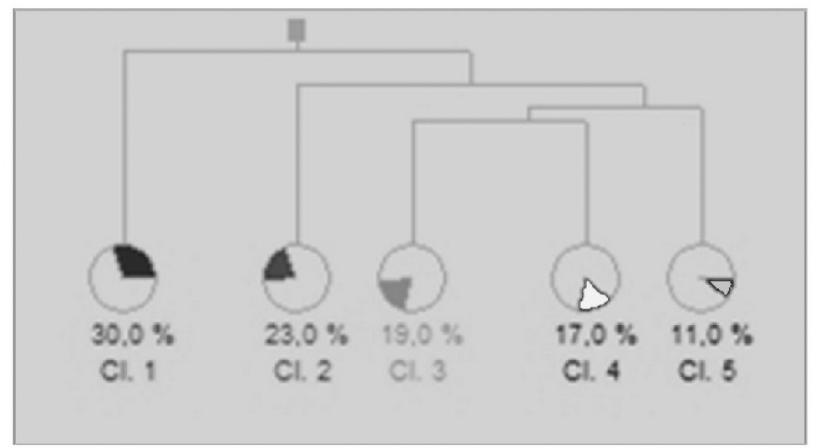

Figure 8. Classes dégagées par ALCESTE dans le corpus de préfaces et de types d'exercices (FLB)

Cet arbre révèle les distances entre les morceaux de textes qui forment les classes de discours. Les classes dont le vocabulaire est le plus proche sont donc les classes 3 et 4 , reliées ensuite à la classe 5 , puis à la classe 2 . Toutes ces classes figurent sur la partie droite de la classification descendante, et se voient donc opposées à la classe 1 , seule classe composant la branche gauche de cette classification.

On observe que cette première classe (en rouge), nettement détachée des autres, est très fortement représentée. Elle rassemble des termes définissant les objectifs et principes directeurs de la formation : «compréhension, bases, compétence, autonomie, maîtrise, connaissance, action, apprentissage, formation, etc. ». Les dates 1972 et 1990 apparaissent significatives. Or celles-ci correspondent d'une part aux manuels parmi les plus anciens du corpus et de l'autre à la période ayant vu une focalisation sur une structuration du secteur et de ses objectifs et méthodologies d'enseignement. On trouve donc là encore des signes indiquant que les objectifs et principes de formation ont des origines différentes des moyens de leur mise en œuvre.

Les quatre autres classes, formant la branche droite de l'arbre, définissent des actions d'apprentissage. Les années significatives mises en évidence au sein de cette branche recouvrent l'ensemble de la période concernée par notre corpus.

Ainsi la deuxième classe rassemble-t-elle des termes caractéristiques des exercices de phonétique, de morphosyntaxe ou de vocabulaire : « repérer, féminin, pluriel, masculin, conjuguer, singulier, mettre, réponse, question, phonème... ». Ces termes ne sont en corrélation avec aucun ensemble ou type d'exercices particuliers, mais ils correspondent aux termes techniques issus des exercices de l'enseignement scolaire. 
La troisième classe, quant à elle, regroupe des termes caractéristiques des exercices de reproduction de l'écrit pour les plus petits niveaux. Les termes décrivent l'action des apprenants autour d'activités d'écriture et de manipulation de texte : « recopier, chiffre, donnée, relever, contrainte, reproduire, schématiser, tracer, lettre, graphies, communication, détaillées.... ». Cette classe est caractérisée par l'ensemble Livre de français pour adultes immigrés et sa préface, les ensembles DILF A1.1 et La lecture Début de l'insertion, ainsi que les exercices de Formation lecture. Les années les plus significatives sont 1975, 1986, 1989, et 2008.

La quatrième classe, réunit des éléments liés aux exercices de production orale et écrite correspondant à une maîtrise plus élaborée et plus autonome de l'écrit et du langage : "support, donner, trouver, produire, compléter, sujet, énoncé, jeu rôle, résumer, formulaire, débat... ». Elle est associée aux exercices et à l'ensemble pédagogique Expression française, aux exercices de Trait d'Union et aux années 1974 et 2001.

Enfin, la cinquième classe s'organise autour des activités d'évaluation et d'opérations cognitives, dont la maitrise essentielle au passage des activités de la troisième classe à celles de la quatrième classe : «tableau, double, entrée, logique, chronologique, croissant, décroissant, classer, fréquent, sommative, évaluation, ordre...». Elle est associée aux ensembles pédagogiques et aux exercices du Livre de français pour adultes immigrés et de Lecture et Vie, aux exercices de Trait d'Union et de Maîtriser les écrits du quotidien, ainsi qu'aux années 1975 et 1989.

La question de l'accès à l'écrit traverse largement les classes 3 à 5 . À nouveau, l'enseignement de l'écrit, et surtout de la lecture, semble constituer le socle des activités mises en place par les formateurs. Cette donnée explique très certainement que des références caractéristiques du champ du FLE n'apparaissent pas de façon significative dans le corpus. En effet, le FLE s'adresse avant tout à des apprenants déjà lecteurs-scripteurs dans leur langue première. Il est entendu que ces compétences en littéracie sont transférées lors de l'apprentissage du français comme langue étrangère. Mais l'on voit dans les classes 3 à 5 que les questions significativement associées aux pratiques de formation du secteur qui nous intéresse sont centrées sur les apprentissages premiers de l'écrit (copier, repérer...). En conséquence de quoi, le FLE ne peut pas entièrement répondre aux besoins des enseignants et c'est le secteur du FLM et plus spécifiquement de l'enseignement scolaire (classe 2) qui est plus fortement convoqué pour l'agir professionnel.

Nous avions fait l'hypothèse, au départ, qu'il y aurait un certain décalage entre les principes pédagogiques exprimés dans les préfaces et leur réalisation 
dans les exercices. Le résultat de l'analyse sémantique via Alceste est saisissant, car il indique une dissociation forte entre les principes pédagogiques exprimés dans les préfaces (classe 1) et la plupart des types d'exercices et d'activités (classes 3 à 5). Il montre également des pôles qui s'organisent non pas en méthodologies d'enseignement constituées et, éventuellement, distinctes, mais plutôt un corps de principes d'action cohérents dans leur ensemble et sur toute la période du corpus et qui se réaliseraient à travers des types d'exercices plutôt caractéristiques de l'enseignement scolaire (classe 2).

\section{5 - Conclusion}

Au travers des matériels pédagogiques de la formation linguistique de base, nous avons découvert un secteur professionnel en cours de construction et dont les tiraillements identitaires sont exprimés par les outils qu'il utilise au quotidien. Les matériels pédagogiques constituent un corps cohérent d'ouvrages, dont le cœur est spécifiquement destiné aux apprenants de la formation linguistique de base : des adultes peu ou pas francophones et/ ou peu ou pas familiarisés avec l'écrit. Ce corps cohérent de matériels utilisés en formation repose sur un corps relativement stable de principes d'action, largement issus des années 1980 à 1990, marqués par la forte influence du secteur de la formation continue et de la formation professionnelle. On voit là un secteur professionnel dont les principes pédagogiques constituent un socle commun. Mais celui-ci cherche encore les moyens de s'actualiser pleinement dans des actions pédagogiques spécifiques à la formation d'adultes. En effet, une grande partie du rapport à la mise en œuvre de principes d'action semble devoir être construite, car leur réalisation passe par des types d'activités caractéristiques d'abord de l'enseignement scolaire pour l'enseignement de l'écrit, puis du FLE pour l'enseignement de l'oral. C'est ainsi qu'aux deux secteurs définis dans notre introduction : la formation continue et le FLE, il faut ajouter celui de l'enseignement scolaire parmi les influences notables de l'agir professionnel des formateurs de la formation linguistique de base des résidents étrangers.

La récente réforme du Français Langue d'Intégration, s'appuyant sur un référentiel métier et des critères de qualité pour les structures de formation s'appuie, de fait, sur la cohérence existante dans le discours du secteur et semble l'accompagner vers la constitution d'un véritable groupe professionnel : aux valeurs communes et aux principes fondateurs déjà établis, il vient ajouter un diplôme et une certification des compétences professionnelles ainsi que des garanties de qualité pour les apprenants et commanditaires. Or ces trois éléments constituent pour bon nombre d'auteurs (Abbalea, 2000, 
2005 ; Bourdoncle, 2000) tout ou partie des critères définitoires d'une profession constituée. Reste à savoir si cette stabilisation du groupe professionnel aura un impact sur son agir professionnel et ce sont les recherches des années à venir qui pourront nous le dire.

\section{Références}

Abbalea F. (2000). «Travail social et intervention sociale : de la catégorisation à l'identité ». Recherches et prévisions, $\mathrm{n}^{\circ}$ 62, p. 71-81.

Abbalea F. (2005). «La professionnalisation inachevée des assistantes maternelles ». Recherches et Prévisions, n 80, p. 55-65.

Adami H. (2005). « Les faux jumeaux didactiques ». Le francais dans le monde, $\mathrm{n}^{\circ} 339$, p. 23-25.

Adami H. (2008). " Le rôle de la littératie dans le processus d'acculturation des migrants ». Conseil de l'Europe.

Adami H. (2009). La formation linguistique des migrants. Paris : CLE.

Barthélémy F. (2007). Professeur de FLE : historique, enjeux et perspectives. Paris : Hachette.

Beacco J. C., Ferrari M., de Lhote G. et al. (2005). Nivean A1.1 pour le francais (Publics adultes peu francophones, scolarisés, peu ou non scolarisés - Référentiel et certification (DILF) pour les premiers acquis en français). Paris : Didier.

Bergère A. (2008). "Formation linguistique des adultes : un état des lieux des ressources pour les nouveaux formateurs ». Accueillir, $\mathrm{n}^{\circ} 248$, p. 49-50.

Bertoletti M. C., Dahlet P. (1984). «Manuels etmatériels scolaires pour l'apprentissage du FLE - Ébauche d'une grille d'analyse ». Le français dans le monde, $\mathrm{n}^{\circ} 186$, p. 5563.

Blot B., Mariet F., Porcher L. (1978). Pour la formation des travailleurs migrants. Paris : Didier.

Bourdoncle R. (2000). "Autour des mots "Professionnalisation, formes et dispositifs" ", Recherche et formation, n³5, p.117-132.

Castells M. (1999). L'ère de l'information. Tome II. Le pouvoir de l'identité. Paris : Fayard.

Cordier-Gauthier C. (2002). "Les éléments constitutifs du discours du manuel ». Revue de didactologie des langues-cultures, $\mathrm{n}^{\circ} 125, \mathrm{p}$. 25-36.

Coste D., Courtillon J., Frenczi V. et al. (1976). Un niveau-seuil. Paris : Hatier-Didier.

Coulbaut-Lazzarini, A. (2012). Le secteur de la formation linguistique de base en France: une étude exploratoire. Paris : VA Savoirs.

Cuq J.-P. (2003). Dictionnaire didactique langue étrangère et seconde. Paris : CLE/ ASDIFLE. 
Cuq J.-P., Gruca I. (2003). Cours de didactique du français langue étrangère. Grenoble : PUG.

Dubar C., Tripier P. (2005) [1 ${ }^{\text {re }}$ éd. 1998]. Sociologie des professions. Paris : Armand Colin.

Germain C. (1993). Évolution de l'enseignement des langues : 5000 ans d'histoire. Paris : CLE.

Haut conseil à l'intégration (2002). Lesparcours d'intégration. Paris : La Documentation française.

Leclercq V. (2004). « La professionnalisation des formateurs impliqués dans la formation de base. Un processus inachevé ». Ville-École-Intégration Enjeux, $\mathrm{n}^{\circ} 136$, p. $187-202$.

Leclercq V. (2010). «La formation linguistique des migrants des années 1960 aux années 1980 ». Éducation Permanente, n 183, p. 173-187.

Lehoussel J., Bergère A. (2010). Description du corpus de référence pour les phases 1 et 2. In Publications du projet MALIN. Paris : Association VA Savoirs., consultable sur http:/ /www.va-savoirs.org.

Observatoire national de la lecture (2003). Le manuel de lecture au CP. Scéren / Savoir Livre

Observatoire national de la lecture (2007). L'enseignement de la lecture et l'observation des manuels de lecture du CP - Rapport de l'observatoire. ONL.

Perdue C. (dir.) (1984). Second language acquisition by adult immigrants: a field manual. Rowley, Mass.: Newbury House Publishers.

Porcher L. (dir.) (1982). Identification des besoins langagiers de travailleurs migrants en France. Strasbourg : Conseil de la coopération culturelle / Saint-Cloud : CREDIF.

Porcher L. (1987). Enseigner-diffuser le français : une profession. Paris : Hachette.

Puren C. (2000). «Méthodes et constructions méthodologiques dans l'enseignement et l'apprentissage des langues. » Les Langues modernes, $n^{\circ} 1$, p. 68-70.

Puren C. (2001). Outils et méthodologie d'analyse des manuels de langue. ILIAD.

Roure H., Reinert M. (1993). « Analyse d'un entretien à l'aide d'une méthode d'analyse lexicale ». JADT 1993. Paris : ENST.

Vezinat N. (2010). «Une nouvelle étape dans la sociologie des professions en France ». Sociologie, vol. $1, \mathrm{n}^{\circ} 3$.

Vicher A. (dir.) (2011). Référentiel FLI Français langue d'intégration. Paris : Ecrimed. 


\section{Annexe}

Matériels pédagogiques

pour la FLB d'éditeurs spécialiste FLE de fréquence 3 à 9 dans le recensement

\begin{tabular}{|c|c|c|c|c|c|c|c|c|c|c|}
\hline 莺 & 贡 & 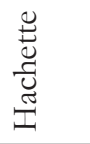 & $\begin{array}{l}0 \\
2 \\
2\end{array}$ & $\begin{array}{l}0 \\
2 \\
2\end{array}$ & 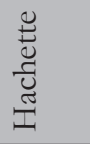 & 节 & 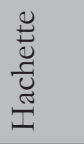 & 苛 & 펍 & $\stackrel{I}{3}$ \\
\hline 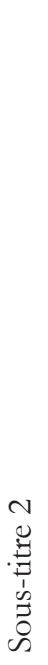 & 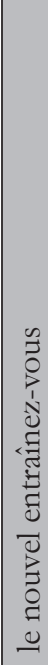 & 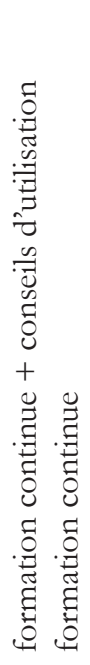 & & 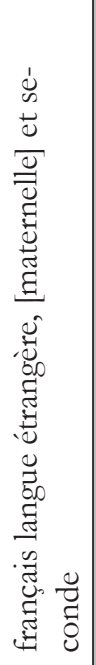 & 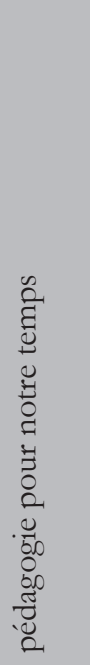 & 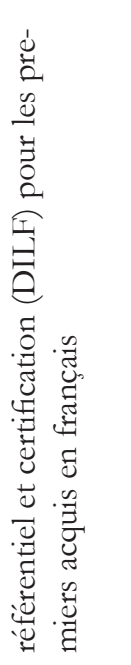 & 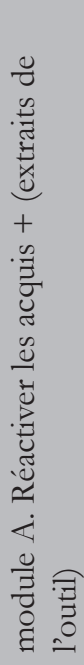 & & 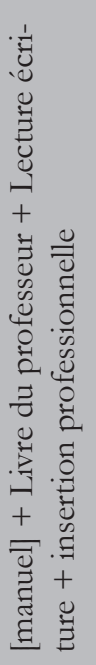 & 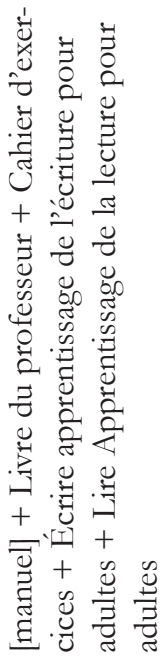 \\
\hline 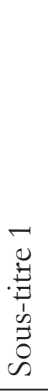 & 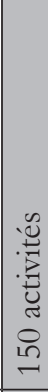 & 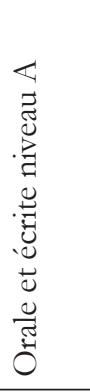 & 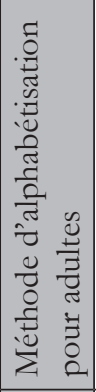 & 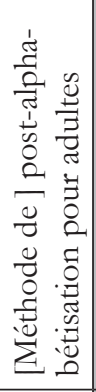 & 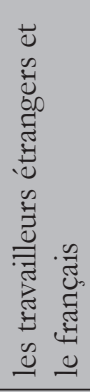 & 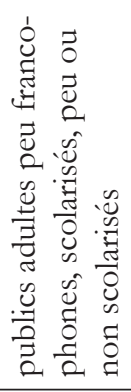 & 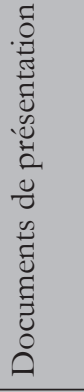 & & 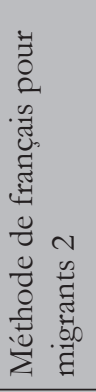 & 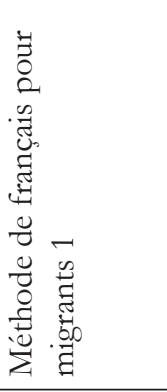 \\
\hline$\underset{\Xi}{\Xi}$ & 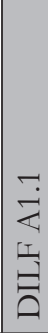 & 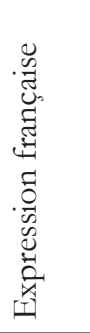 & 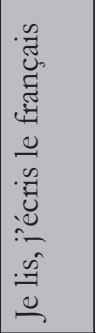 & 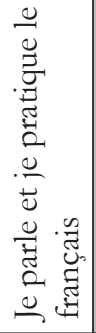 & 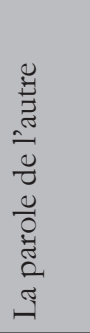 & 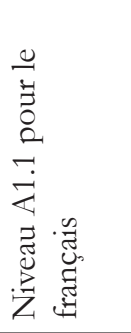 & 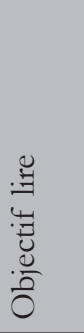 & 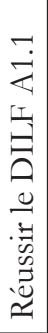 & 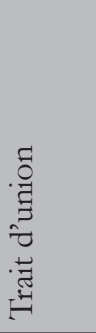 & 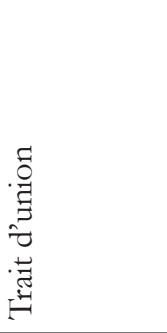 \\
\hline $\begin{array}{l}\text { J } \\
\stackrel{0}{*}\end{array}$ & \& & $\stackrel{+}{\stackrel{+}{2}}$ & ช్ & 号 & $\stackrel{\hat{a}}{\rightleftharpoons}$ & 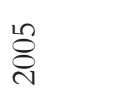 & $\stackrel{+}{2}$ & 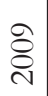 & 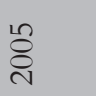 & $\underset{\sim}{\stackrel{్}{్}}$ \\
\hline
\end{tabular}

